\title{
CD4 count at antiretroviral therapy initiation and the risk of loss to follow-up: results from a multicentre cohort study
}

\author{
Anna Grimsrud ${ }^{1}$, Morna Cornell ${ }^{1,2}$, Michael Schomaker ${ }^{2}$, Matthew P Fox ${ }^{3,4,5}$, Catherine \\ Orrell $^{6}$, Hans Prozesky ${ }^{7,8}$, Kathryn Stinson ${ }^{2,9}$, Frank Tanser ${ }^{10}$, Matthias Egger ${ }^{11}$, and \\ Landon Myer ${ }^{1,2}$ for the International Epidemiologic Databases to Evaluate AIDS Southern \\ Africa Collaboration (IeDEA-SA)
}

${ }^{1}$ Division of Epidemiology and Biostatistics, School of Public Health \& Family Medicine, University of Cape Town, Cape Town, South Africa ${ }^{2}$ Centre for Infectious Disease Epidemiology \& Research, School of Public Health \& Family Medicine, University of Cape Town, Cape Town, South Africa ${ }^{3}$ Center for Global Health \& Development, Boston University, Boston, Massachusetts, USA ${ }^{4}$ Department of Internal Medicine, Faculty of Health Sciences, Health Economics and Epidemiology Research Office, School of Clinical Medicine, University of the Witwatersrand, Johannesburg, South Africa ${ }^{5}$ Department of Epidemiology, Boston University School of Public Health, Boston, Massachusetts, USA ${ }^{6}$ Faculty of Health Sciences, Desmond Tutu HIV Centre, Institute for Infectious Diseases and Molecular Medicine, University of Cape Town, Cape Town, South Africa ${ }^{7}$ Division of Infectious Diseases, Department of Medicine, University of Stellenbosch, Cape Town, South Africa ${ }^{8}$ Tygerberg Academic Hospital, Cape Town, South Africa ${ }^{9}$ Médecins Sans Frontières, Cape Town, South Africa ${ }^{10}$ Africa Centre for Health and Population Studies, University of KwaZulu-Natal, Mtubatuba, South Africa ${ }^{11}$ Division of International and Environmental Health, Institute of Social and Preventive Medicine (ISPM), University of Bern, Bern, Switzerland

\section{Abstract}

Correspondence to Dr Anna Grimsrud, Division of Epidemiology \& Biostatistics, School of Public Health \& Family Medicine, University of Cape Town, Anzio Road, Observatory, Cape Town 7925, South Africa; agrimsrud@ gmail.com.

These data were presented at the 8th IAS Conference on HIV Pathogenesis, Treatment \& Prevention (IAS 2015).

Contributors MPF, CO, HP, KS, and FT established/maintained the cohorts and provided data. AG analysed the data and wrote the first draft of the manuscript. AG, MS, and LM contributed to the plan of analysis and to the writing of the report, and interpreted the data. All the authors commented on draft manuscripts and approved the final version.

Competing interests None declared.

Ethics approval Human Research Ethics Committee in the Faculty of Health Sciences at the University of Cape Town and the Human Research Ethics Committee at the University of Bern.

Provenance and peer review Not commissioned; externally peer reviewed.

Data sharing statement Data are accessible in principle by applying for access directly from each contributing cohort. The IeDEASA data set is a multisite collaboration and this analysis combined data from different sites. Therefore, data are not available from the lead author as ownership of the data is retained at the site. Each site has approval from its own local Institutional Review Board to collect routine data on patients and to transfer these data anonymously to the IeDEA-SA data centre for use in collaborative analyses. Interested readers may contact the site investigators to request data. 
Background-Antiretroviral therapy (ART) initiation is now recommended irrespective of CD4 count. However data on the relationship between CD4 count at ART initiation and loss to followup (LTFU) are limited and conflicting.

Methods-We conducted a cohort analysis including all adults initiating ART (2008-2012) at three public sector sites in South Africa. LTFU was defined as no visit in the 6 months before database closure. The Kaplan-Meier estimator and Cox's proportional hazards models examined the relationship between CD4 count at ART initiation and 24-month LTFU. Final models were adjusted for demographics, year of ART initiation, programme expansion and corrected for unascertained mortality.

Results-Among 17038 patients, the median CD4 at initiation increased from 119 (IQR 54180) in 2008 to 257 (IQR 175-318) in 2012. In unadjusted models, observed LTFU was associated with both CD4 counts $<100$ cells $/ \mu \mathrm{L}$ and CD4 counts 2300 cells $/ \mu \mathrm{L}$. After adjustment, patients with CD4 counts 2300 cells/ $\mu \mathrm{L}$ were 1.35 (95\% CI 1.12 to 1.63 ) times as likely to be LTFU after 24 months compared to those with a CD4 150-199 cells/ $\mu \mathrm{L}$. This increased risk for patients with CD4 counts $>300$ cells $/ \mu \mathrm{L}$ was largest in the first 3 months on treatment. Correction for unascertained deaths attenuated the association between CD4 counts $<100$ cells $/ \mu \mathrm{L}$ and LTFU while the association between CD4 counts $\$ 300$ cells/ $\mu \mathrm{L}$ and LTFU persisted.

Conclusions-Patients initiating ART at higher CD4 counts may be at increased risk for LTFU. With programmes initiating patients at higher CD4 counts, models of ART delivery need to be reoriented to support long-term retention.

\section{Introduction}

Access to antiretroviral therapy (ART) has improved considerably in the past decade. By the end of 2013, 12.9 million people globally were receiving ART. ${ }^{1}$ Programmes have increased in size and expanded access with patients initiating ART at higher CD4 counts. In all regions, median CD4 counts at ART initiation are increasing. ${ }^{2}$

Increases in CD4 counts at ART initiation reflect progressive changes in WHO guidelines. Prior to 2010, ART was recommended for adults with CD4 counts below 200 cells $/ \mu \mathrm{L}$ irrespective of WHO clinical stage. ${ }^{3}$ The CD4 threshold was changed in 2010 to 350 cells/ $\mu \mathrm{L}^{4}$ and raised further in the 2013 guidelines to include all patients with a CD4 count of 500 cells $/ \mu \mathrm{L}$ or less. ${ }^{5}$ In September 2015 , the WHO announced ART should be initiated in all people living with HIV at any CD4 count. ${ }^{6}$ The global trend towards earlier initiation of ART is the result of advances in science, improvements in ART drugs and developments in the practice of HIV care. ${ }^{7}$

Despite the potential benefits of earlier ART initiation, its impact on patient behaviour and resulting loss to follow-up (LTFU) is not well understood. LTFU in ART programme represents a considerable challenge and removing the CD4 threshold increases the number of eligible patients for ART. ${ }^{8-10} \mathrm{~A}$ critical obstacle to assessing associations with LTFU is determining whether a patient considered LTFU is truly lost or an unascertained death. A valid measure of LTFU is particularly important when assessing the association between CD4 and LTFU since lower CD4 counts are related to mortality. ${ }^{1112}$ The limited data available on the relationship between CD4 counts at ART initiation and LTFU is conflicting. 
In previous research, higher CD4 counts are associated with both an increased ${ }^{1314}$ and decreased risk of LTFU. ${ }^{1516}$ Given this conflicting evidence, the need to assess adherence and retention in people initiating ART at higher CD4 counts has been highlighted. ${ }^{5}$

We investigated the relationship between CD4 counts at ART initiation and LTFU in the first 24 months on treatment among adults initiating ART between 2008 and 2012 in the South African cohorts of the International epidemiologic Databases to Evaluate AIDS-Southern Africa (IeDEA-SA) collaboration. We hypothesised that after adjustment for individual (age, sex, year of ART initiation) and programme (cohort size, rate of expansion) factors, patients initiating ART at higher CD4 counts may be at an increased risk of LTFU.

\section{Methods}

\section{Study design, population and eligibility criteria}

We conducted a multicentre, retrospective cohort analysis using data from the IeDEA-SA collaboration. The collaboration has been described in detail previously. ${ }^{1718}$ Briefly, patients were included in the analysis if they were ART naïve, 16 years of age or older, not pregnant at ART initiation, initiated ART in 2008 or later, and had a CD4 count measure available at ART initiation. Analysis was restricted to patients who had a minimum of 6 months of follow-up and outcomes were restricted to the first 24 months of treatment. For the main analysis, only patients with a recorded South African civil identification (ID) number were included and thus our main analysis included data from three public sector sites (Hlabisa (cohort 1), Khayelitsha (cohort 2), Themba Lethu (cohort 3)) providing ART free of charge to adults in three South African provinces (Gauteng, Kwa-Zulu Natal and the Western Cape).

Data from an additional two cohorts (Gugulethu and Tygerberg) that did not collect IDs were included in the sensitivity analyses.

\section{Variables and definitions}

At ART initiation, individual demographics (sex and age) and measures of disease severity (CD4 count and WHO stage) were assessed. Two variables were generated to quantify programme expansion: programme size and the rate of programme scale up. For each cohort, the number of ART patients receiving care at the end of the calendar year of ART initiation was defined as a measure of programme size. ${ }^{10}$ The rate of programme scale up was estimated as the rank of the patient divided by the number of months that the programme had been providing ART. For example, the 200th patient enrolling in the 10th month of the programme would have a rate of scale up of $20(200 / 10) .{ }^{10}$

Patients were defined as LTFU if they were not known to be dead or transferred out, and had no visit in the 6 months before the database closure. The date of LTFU was defined as the date of last contact. For patients who had initiated ART and did not return, 1 day of followup was added so that they would be included in the survival analysis. ${ }^{19}$ Patients who were dead or transferred out were censored at the date of death or transfer. 
Corrected estimates of LTFU were made through linkage by ID within the National Population Register (NPR) of the South African Department of Home Affairs. Patients previously defined as LTFU were reclassified as dead if they had a date of death in the NPR within 3 months of their date of LTFU. ${ }^{20}$ Corrected estimates, therefore, reflect incorporating data from the NPR, 'corrected LTFU', whereas uncorrected estimates are based on participant attendance, 'observed LTFU'.

\section{Data analysis}

Patient characteristics at ART initiation were compared by CD4 count categories at ART initiation and reported with appropriate summary statistics (proportions, medians and IQR). The Kaplan-Meier (KM) estimator was used to obtain estimates of the proportion of those LTFU (observed or corrected) and reported every 6 months. KM curves were plotted and presented by CD4 levels at ART initiation.

To assess the association between CD4 count at ART initiation and the risk of LTFU, a series of Cox's proportional hazards regression models were fitted. CD4 count at ART initiation was modelled as a categorical variable in 50 cells $/ \mu \mathrm{L}$ increments up to $>300$ cells/ $\mu \mathrm{L}$. The reference category used was a CD 4 count between 150 and 199 cells $/ \mu \mathrm{L}$ to facilitate examination of possible trends at lower and higher CD4 counts. All models were stratified by cohort. Separate models were used to assess observed and corrected LTFU. The proportional hazards of LTFU were modelled overall (0-24 months) and separately for 0-3, 3-12 and 12-24 months after ART initiation. Schoenfeld residuals were used to confirm the proportional hazards assumption. Adjusted models included other variables at ART initiation: year of ART initiation, sex, age, cohort size and rate of scale up. In the main analysis, WHO status was excluded because of the large amount of missing data (36\% missing).

A series of sensitivity analyses were conducted to explore the impact of missing data and non-differential censoring. To address missing IDs, inverse probability weighting was done whereby patients with IDs who were not alive and in care were weighted to represent all patients who were not alive and in care. ${ }^{21-24}$ Weighting of patients with IDs was determined as the probability of being in a cohort with IDs multiplied by the modelled probability of having a recorded ID (based on cohort, year of ART initiation, final outcome and CD4 count at ART initiation). Multiple imputation using chained equation methods ${ }^{25}$ was used to impute missing CD4 counts at ART initiation. We multiply imputed CD4 counts (5 times) and the imputation models included the same variables as the Cox models. Competing risk regression was employed to model time to LTFU taking the competing event of death into account. ${ }^{10}$

Data were analysed using STATA V.13.0 (STATA Corporation, College Station, Texas, USA). Ethical approval was received from the relevant institutions at each of the sites and the University of Cape Town Human Research Ethics Committee. 


\section{Results}

The main analysis included 17038 adults who initiated ART between January 2008 and June 2012 in one of three cohorts (table 1). Median CD4 at ART initiation increased with each successive calendar year from 119 cells $/ \mu \mathrm{L}$ in 2008 (IQR 54-180) to 257 cells $/ \mu \mathrm{L}$ in 2012 (IQR 175-318). The proportion of patients in WHO stage IV at ART initiation was $25 \%, 17 \%, 9 \%, 8 \%$ and $17 \%$ for patients in CD4 groups <50, 50-99, 100-199, 200-299 and 2300 cells $/ \mu \mathrm{L}$, respectively. The median programme size by individuals was almost 10 000 patients (median 9846, IQR 7588-11 074) with an average of 160 new patients initiating treatment every month (median 167, IQR 132-192).

\section{Proportion of LTFU}

A cumulative total of $6 \%, 10 \%, 14 \%$ and $17 \%$ of patients were observed to be LTFU at 6 , 12,18 and 24 months, respectively. Observed proportions of LTFU differed by CD4 count at ART initiation (figure 1A). The highest proportion of observed LTFU was seen in patients with CD4 counts at ART initiation $<100$ or $\geq 300$ cells $/ \mu \mathrm{L}$, and the lowest proportions in patients initiating ART between 100 and 200 cells/ $\mu \mathrm{L}$. Rates of observed LTFU also varied by cohort with 12-month observed LTFU ranging from $7 \%$ to $13 \%$ and 24-month observed LTFU from $12 \%$ to $21 \%$. Consistent across all cohorts were high proportions of observed LTFU in patients with CD4 counts below 50 cells $/ \mu \mathrm{L}$. Proportions of observed LTFU were highest in patients with $<50$ and 2300 cells/ $\mu \mathrm{L}$ CD4 counts at ART initiation when stratified by year of ART initiation (results not shown).

After accounting for unascertained deaths, LTFU decreased over time with corrected cumulative LTFU estimated at 5\%,8\%,11\% and $14 \%$ at $6,12,18$ and 24 months, respectively (figure 2). Correcting for deaths accounted for a relative reduction in LTFU of between 16\% (24 months) and 30\% (6 months) (results not shown). Corrected LTFU differed by CD4 count at ART initiation (figures 1B and 2). Proportions of corrected LTFU appeared similar among all patients initiating ART with CD4 counts $<200$ cells $/ \mu \mathrm{L}$, and were highest in patients initiating ART at a CD4 count 2300 cells $/ \mu \mathrm{L}$ (figure 1B). This was observed in all cohorts. As with observed LTFU, corrected LTFU varied by cohort. 12month LTFU ranged from $7 \%$ to $10 \%$ and 24-month LTFU from $12 \%$ to $18 \%$ (results not shown).

\section{Risk of LTFU}

Overall, an increased risk of observed LTFU was seen in patients initiating ART with $<100$ cells $/ \mu \mathrm{L}$ and CD4 counts 2300 cells/ $\mu \mathrm{L}$ compared to patients with a CD4 count between 150 and 199 cells/ $\mu \mathrm{L}$ (figure 3 and online supplementary appendix 1). After adjusting for demographics, year of ART initiation, programme size, and rate of scale up, the increased risk of observed LTFU persisted for those with a CD4 count $<100$ and 2300 cells $/ \mu \mathrm{L}$ at ART initiation compared to patients with a CD4 count between 150 and 199 cells $/ \mu \mathrm{L}$ (table 2 and online supplementary appendix 1 ).

Patients initiating ART with CD4 counts 2300 cells $/ \mu \mathrm{L}$ were at an increased risk of being LTFU over 24 months of ART (table 2). Patients initiating ART with a CD4 count $\geq 300$ 
cells/ $\mu \mathrm{L}$ were $35 \%$ more likely to be LTFU after 24 months of ART compared to patients with a CD4 count between 150 and 199 cells/ $\mu \mathrm{L}$ at ART initiation (adjusted HR 1.35, 95\% CI 1.12 to 1.63). The risk of LTFU was largest in the first year on ART (table 2). After 12 months of treatment, no significant association was observed between CD4 count at ART initiation and corrected LTFU. The increased risk of observed LTFU among patients with lower CD4 counts at ART initiation was attenuated by unascertained deaths and did not persist in crude or adjusted models of corrected LTFU (figure 3 and table 2).

The association between CD4 count at ART initiation and LTFU was assessed separately for each of the three cohorts (see online supplementary appendix 2). The results in cohorts 1 and 2 were very similar to the overall associations observed between CD4 count and LTFU. For cohort 3, patients with CD4 counts $<100$ cells $/ \mu \mathrm{L}$ and with CD4 counts between 200 and 249 cells/ $\mu \mathrm{L}$ had a decreased risk of LTFU compared to patients initiating ART with CD4 counts between 150 and 199 cells/ $\mu \mathrm{L}$. In this cohort, a limited number of patients initiated treatment at CD4 counts above 200 cells/ $\mu \mathrm{L}(\mathrm{n}<500)$ and later than $2009(\mathrm{n}<50)$. Median CD4 count in cohort 3 was 105 compared to 142 and 151 in the other two cohorts, respectively.

Subgroup analyses were done to assess the association between CD4 count at ART initiation and LTFU by year of ART initiation, sex and age (see online supplementary appendix 3). In each year of ART initiation, an increased hazard of LTFU was observed among patients initiating ART with a CD4 count of 2300 cells $/ \mu \mathrm{L}$ compared to patients initiating ART between 150 and 199 cells $/ \mu \mathrm{L}$. In final models, women were 35\% more likely (aHR 1.35 , 95\% CI 1.09 to 1.68 ) and men were $24 \%$ more likely (aHR $1.24,95 \%$ CI 0.84 to 1.82 ) to be LTFU if they initiated ART with a CD4 count 2300 cells/ $\mu \mathrm{L}$ compared to patients initiating ART between 150 and 199 cells $/ \mu \mathrm{L}$. An increased risk of LTFU was observed in patients 25 years and older with a CD4 count 2300 cells $/ \mu \mathrm{L}$ compared to patients initiating ART for CD4 counts between 150 and 199 cells/ $\mu \mathrm{L}$. No association between a CD4 count 2300 cells/ $\mu \mathrm{L}$ and LTFU was observed in patients $<25$ years of age (HR $0.99,95 \% \mathrm{CI} 0.61$ to 1.58 ).

Of the five cohorts assessed for eligibility, two cohorts did not collect IDs. There were 26 466 patients with missing data (CD4 counts were missing in 5457 patients at ART initiation, 4828 did not have a recorded ID, and 16161 did not have a CD4 count or recorded ID). Characteristics of patients with and without IDs are summarised in online supplementary appendix 4. In sensitivity analyses accounting for missing data, the association between higher CD4 count at ART initiation and increased risk of early LTFU persisted, similar to the main results. When inverse probability weighting was used to account for missing IDs, patients with a CD4 count 2300 cells/ $\mu \mathrm{L}$ at ART initiation were $55 \%$ more likely to be LTFU in the first 3 months of ART (95\% CI 0.94 to 2.55) (see online supplementary appendix 5). This association is slightly reduced compared to the results in the main analysis (aHR 1.68, 95\% CI 1.15 to 2.45) (table 2). Similarly, in multiple imputation models including patients with missing CD4 counts, a CD4 count 2300 cells $/ \mu \mathrm{L}$ was associated with a 50\% increase in LTFU in the first 3 months of ART (95\% CI 1.04 to 2.18) (see online supplementary appendix 6 ). The competing risk analysis yielded similar conclusions to the main results (see online supplementary appendix 7). 


\section{Discussion}

Our analyses demonstrate that patients initiating ART at CD4 counts $\geq 300$ cells/ $\mu \mathrm{L}$ are at an increased risk of LTFU in the first 24 months on ART. The increased risk of LTFU among patients with higher CD4 counts at ART initiation was observed both with and without correction for unascertained deaths and persisted after adjustment for individual factors and measures of programme expansion. The greatest increase in risk of LTFU for patients initiating with higher CD4 counts was in the first year on treatment. While low CD4 count at ART initiation was associated with increased risk LTFU, correction for unascertained deaths removed this association.

Our finding that CD4 counts 3300 cells/ $\mu \mathrm{L}$ are associated with higher risk of LTFU is aligned with previous findings ${ }^{1314}$ from smaller, individual studies. In Uganda, a tracing study of patients LTFU found higher rates of LTFU among those with CD4 counts $\geq 250$ cells $/ \mu \mathrm{L}$. CD 4 counts 2250 cells $/ \mu \mathrm{L}$ were also associated with treatment interruptions. ${ }^{26}$ Conversely, other studies have found higher CD4 counts were associated with reduced LTFU. ${ }^{1627}$ However, limited data were available on patients with CD4 counts 2300 cells $/ \mu \mathrm{L}$ and LTFU was not corrected for unascertained deaths.

We can speculate as to why patients who initiate ART at higher CD4 counts may be at an increased risk of being lost to care. ART services have been designed to support acutely ill HIV patients with lower CD4 counts. Patients who initiate ART at higher CD4 counts without an illness experience are not catered for in the services, and they may not experience any immediate benefits of ART and thus, disengage from care early. ${ }^{13}$ The association between CD4 counts of 2300 cells $/ \mu \mathrm{L}$ and risk of LTFU was highest in the first year on treatment, especially in the first 3 months. This suggests interventions are needed to change how patients with higher CD4 cell counts are initiated onto ART. Given the range of CD4 counts at which patients are now initiating ART, models of ART provision that triage patients at initiation based on CD4 count should be considered to provide more tailored support. In addition, pretherapy counselling should be updated to address the distinct challenges faced by patients initiating treatment at higher CD4 counts. ${ }^{28}$ Beyond ART initiation, patients with higher CD4 counts at ART initiation may benefit from receiving ART outside of traditional facility-based models by accessing ART in community-based models of care.

Our finding that patients initiating ART with higher CD4 counts are at greater risk of being LTFU is relevant as countries align national ART guidelines with the 2015 WHO guidelines. ${ }^{56}$ Given the advances in ART drugs and the evidence that earlier ART can benefit both the individual and the population; the question is less about when patients should initiate ART, but how. It is paramount that the change in ART eligibility criteria is accompanied by health system adaptations. We need to adapt where and how patients with higher CD4 counts are initiated into ART given the increased risk of LTFU in the first year on treatment. We are reliant on a limited number of service delivery models that may not provide the most appropriate care for our evolving ART programmes. Furthermore, innovative models of care are needed to improve long-term retention of patients. It is also apparent that we are going to need a variety of models; models that can coexist and have 
strong referral mechanisms to meet the different needs of increasingly heterogeneous populations accessing ART. ${ }^{29}$ Options for populations who currently have inequitable access to ART, including men, older populations, and underserved populations, are also needed.

We are uniquely positioned to assess the association between a range of CD4 counts and LTFU. With data from multiple cohorts, we could look at more patients at higher CD4 thresholds than previous research. The challenge faced by many ART cohort analyses of differentiating risk factors for corrected LTFU from risk factors for unascertained deaths is overcome through linkage with the NPR. The quality of the NPR is very high with nearly $90 \%$ of adult deaths captured. ${ }^{30} \mathrm{~A}$ valuable finding provided through linkage was that no significant association between lower CD4 counts and corrected LTFU was observed. This suggests that the association in uncorrected models was an artefact of unascertained mortality. That the association between CD 4 counts $2300 \mathrm{cells} / \mu \mathrm{L}$ and LTFU remained after linkage gives weight to our findings. Novel to our findings is that the association persisted after adjusting for measures of programme expansion. Therefore, increases in LTFU are not necessarily the result of deteriorating quality of care as ART programmes have expanded. ${ }^{31}$ Changes to the profile of patients at ART initiation, including a greater number of patients at higher CD4 cell counts without an illness experience, are related to increased LTFU, a result of the model of ART delivery not being appropriate for all patients accessing ART.

Our study should be considered in light of a number of limitations. Data were missing on a number of key variables, including CD4 counts at ART initiation and South African civil ID numbers. We chose to exclude patients with missing CD4 levels postulating that missing data was likely to be related to administrative and clerical errors and is, therefore, likely to be missing completely at random (ie, not related to particular covariates). In sensitivity analyses, models with imputed missing CD4 counts and weighted for missing IDs yielded consistent findings to the main results. Previous analyses also found patients with and without IDs were comparable. ${ }^{23}$ We also observed differences in the relationship between CD4 count at ART initiation and the risk of LTFU when assessed by cohort. Although we were unable to explore explanations for such differences, it is plausible that these may be due to disparities between sites, such as rate and size of programme scale up, and therefore variations in the quality of care provided. Data on patients with CD4 counts 2300 cells/ $\mu \mathrm{L}$ were limited as the change in South African guidelines for initiation of patients with a CD4 count $<350$ cells $/ \mu \mathrm{L}$ was only announced in August 2011. ${ }^{32}$ Therefore, patients with higher CD4 counts in our analysis may have been initiated for other clinical reasons and not be representative of patients initiating ART at higher CD4 counts. In this analysis, $17 \%$ of patients initiating with a CD 4 count 2300 cells $/ \mu \mathrm{L}$ were in WHO stage IV. From a programmatic perspective, our analysis is limited to reporting on LTFU after ART initiation and ignores that a considerable proportion of the patients determined to be LTFU may be treatment interrupters who will re-engage with ART services at a later date. ${ }^{31}$

Our study found that patients who initiate ART with a CD4 count 2300 cells $/ \mu \mathrm{L}$ are at an increased risk of being LTFU after 24 months on treatment compared to patients initiating with a CD 4 count between 150 and 199 cells/ $\mu \mathrm{L}$. Acknowledging the potential benefits of early ART initiation, further research is needed on how to successfully support patients who initiate treatment at higher CD4 counts. This is especially relevant as countries transition to 
'Treat All' offering ART to everyone living with HIV. It is widely acknowledged that the greatest threat to the long-term success of the rapidly expanding ART programmes is ensuring adherence and retention in care. ${ }^{33}$ Given these challenges, now is the time to innovate our health systems to more effectively and efficiently deliver ART.

\section{Acknowledgments}

Funding Support for this study was provided by the US National Institute of Allergy and Infectious Diseases (NIAID) (http://www.niaid.nih.gov/) through the International epidemiological Databases to Evaluate AIDS, Southern Africa (IeDEA-SA), grant number 5U01AI069924-05. Its contents are solely the responsibility of the authors and do not necessarily represent the official views of the NIH. AG receives $\mathrm{PhD}$ funding from the Canadian Institutes of Health Research (CIHR) (http://www.cihr-irsc.gc.ca/e/193.html) and the South African Centre for Epidemiological Modelling and Analysis (SACEMA) (http://www.sacema.com/). MPF is supported by Cooperative Agreement AID 674-A-12-00029 from the USA Agency for International Development (USAID) (http:// www.usaid.gov/). LM is supported by an International Leadership Award from the Elizabeth Glaser Pediatric AIDS Foundation (http://www.pedaids.org/).

\section{References}

1. UNAIDS. The gap report. Geneva: UNAIDS; 2014.

2. World Health Organization. Global update on HIV treatment 2013: results, impact and opportunities. Geneva: World Health Organization; 2013.

3. World Health Organization. Antiretroviral therapy for HIV infection in adults and adolescents: recommendations for a public health approach (2006 revision). Geneva: World Health Organization; 2006.

4. World Health Organization. Antiretroviral therapy for HIV infection in adults and adolescents: recommendations for a public health approach. Geneva: World Health Organization; 2010.

5. World Health Organization. Consolidated guidelines on the use of antiretroviral drugs for treating and preventing HIV infection: recommendations for a public health approach. Geneva: World Health Organization; 2013.

6. World Health Organization. Guideline on when to start antiretroviral therapy and on pre-exposure prophylaxis for HIV. Geneva: World Health Organization; 2015. p. 78

7. Easterbrook PJ, Irvine CJ, Vitoria M, et al. Developing the 2013 WHO consolidated antiretroviral guidelines. AIDS. 2014; 28(Suppl 2):S93-100. [PubMed: 24849484]

8. Cornell M, Grimsrud A, Fairall L, et al. Temporal changes in programme outcomes among adult patients initiating antiretroviral therapy across South Africa, 2002-2007. AIDS. 2010; 24:2263-70. [PubMed: 20683318]

9. Auld AF, Mbofana F, Shiraishi RW, et al. Four-year treatment outcomes of adult patients enrolled in Mozambique's rapidly expanding antiretroviral therapy program. PLoS Med. 2011; 6:e18453.

10. Grimsrud A, Balkan S, Casas EC, et al. Outcomes of antiretroviral therapy over a 10-year period of expansion: a multicohort analysis of African and Asian HIV programs. J Acquir Immune Defic Syndr. 2014; 67:e55-66. [PubMed: 24977472]

11. Boulle A, Bock P, Osler M, et al. Antiretroviral therapy and early mortality in South Africa. Bull World Health Organ. 2008; 86:678-87. [PubMed: 18797643]

12. Brinkhof MW, Boulle A, Weigel R, et al. Mortality of HIV-infected patients starting antiretroviral therapy in sub-Saharan Africa: comparison with HIV-unrelated mortality. PLoS Med. 2009; 6:e1000066. [PubMed: 19399157]

13. Van Cutsem G, Ford N, Hildebrand K, et al. Correcting for mortality among patients lost to follow up on antiretroviral therapy in South Africa: a cohort analysis. PLoS Med. 2011; 6:e14684.

14. Alamo ST, Colebunders R, Ouma J, et al. Return to normal life after AIDS as a reason for lost to follow-up in a community-based antiretroviral treatment program. J Acquir Immune Defic Syndr. 2012; 60:e36-45. [PubMed: 22622076] 
15. HIV Modelling Consortium Treatment as Prevention Editorial Writing Group. HIV treatment as prevention: models, data, and questions - towards evidence-based decision-making. PLoS Med. 2012; 9:e1001259. [PubMed: 22802739]

16. Clouse K, Pettifor A, Maskew M, et al. Initiating antiretroviral therapy when presenting with higher CD4 cell counts results in reduced loss to follow-up in a resource-limited setting. AIDS. 2013; 27:645-50. [PubMed: 23169326]

17. Cornell M, Technau K, Fairall L, et al. Monitoring the South African National Antiretroviral Treatment Programme, 2003-2007: the IeDEA Southern Africa collaboration. S Afr Med J. 2009; 99:653-60. [PubMed: 20073292]

18. Egger M, Ekouevi DK, Williams C, et al. Cohort profile: the international epidemiological databases to evaluate AIDS (IeDEA) in sub-Saharan Africa. Int J Epidemiol. 2012; 41:1256-64. [PubMed: 21593078]

19. Grimsrud AT, Cornell M, Egger M, et al. Impact of definitions of loss to follow-up (LTFU) in antiretroviral therapy program evaluation: variation in the definition can have an appreciable impact on estimated proportions of LTFU. J Clin Epidemiol. 2013; 66:1006-13. [PubMed: 23774112]

20. Cornell M, Schomaker M, Garone DB, et al. Gender differences in survival among adult patients starting antiretroviral therapy in South Africa: a multicentre cohort study. PLoS Med. 2012; 9:e1001304. [PubMed: 22973181]

21. Schomaker M, Gsponer T, Estill J, et al. Non-ignorable loss to follow-up: correcting mortality estimates based on additional outcome ascertainment. Stat Med. 2014; 33:129-42. [PubMed: 23873614]

22. Brinkhof MW, Dabis F, Myer L, et al. Early loss of HIV-infected patients on potent antiretroviral therapy programmes in lower-income countries. Bull World Health Organ. 2008; 86:559-67. [PubMed: 18670668]

23. Boulle A, Schomaker M, May MT, et al. Mortality in patients with HIV-1 infection starting antiretroviral therapy in South Africa, Europe, or North America: a collaborative analysis of prospective studies. PLoS Med. 2014; 11:e1001718. [PubMed: 25203931]

24. Cornell M, Lessells R, Fox MP, et al. Mortality among adults transferred and lost to follow-up from antiretroviral therapy programmes in South Africa: a multicenter cohort study. J Acquir Immune Defic Syndr. 2014; 67:e67-75. [PubMed: 24977471]

25. White IR, Royston P, Wood AM. Multiple imputation using chained equations: issues and guidance for practice. Stat Med. 2011; 30:377-99. [PubMed: 21225900]

26. Adakun SA, Siedner MJ, Muzoora C, et al. Higher baseline CD4 cell count predicts treatment interruptions and persistent viremia in patients initiating ARVs in rural Uganda. J Acquir Immune Defic Syndr. 2013; 62:317-21. [PubMed: 23242160]

27. Ford N, Kranzer K, Hilderbrand K, et al. Early initiation of antiretroviral therapy and associated reduction in mortality, morbidity and defaulting in a nurse-managed, community cohort in Lesotho. AIDS. 2010; 24:2645-50. [PubMed: 20980868]

28. Chaiyachati KH, Ogbuoji O, Price M, et al. Interventions to improve adherence to antiretroviral therapy: a rapid systematic review. AIDS. 2014; 28(Suppl 2):S187-204. [PubMed: 24849479]

29. Holmes C, Pillay Y, Mwango A, et al. Health systems implications of the 2013 WHO consolidated antiretroviral guidelines and strategies for successful implementation. AIDS. 2014; 28(Suppl 2):S231-9. [PubMed: 24849483]

30. Dorrington, R.; Bourne, B.; Bradshaw, D., et al. The impact of HIV/AIDS on adult mortality in South Africa. Cape Town: Burden of Disease Research Unit, Medical Research Council; 2001.

31. Johnson L, Estill J, Keiser O, et al. Do increasing rates of loss to follow-up in antiretroviral therapy programs imply deteriorating patient retention? Am J Epidemiol. 2014; 180:1208-12. [PubMed: 25399412]

32. The Presidency. Republic of South Africa, Statement on the meeting of the South African National AIDS Council (SANAC). 2011. www.thepresidency.gov.za/pebble.asp?relid=4650

33. World Health Organization. Retention in HIV Programmes defining the challenges and identifying solutions: meeting report, 13-15 September 2011. Geneva: 2012. 


\section{What is already known on this subject}

Data on the relationship between CD4 count at the time of initiating antiretroviral therapy and the risk of loss to follow-up are limited and inconsistent. Few studies have large numbers of patients starting antiretroviral therapy at higher CD4 counts. Also, estimates of loss to follow-up can be invalid if there are high rates of unreported deaths. We were able to combine data from multiple sites and correct for unreported deaths through linkage with a death registry, thus having sufficient data to assess the relationship between higher CD4 counts and an accurate measure of loss to follow-up. 


\section{What this study adds}

We now have evidence that patients who have higher CD4 counts when they start antiretroviral treatment may be more likely to be lost to the treatment programme. This finding suggests we should reconsider how health systems can best support patients who are relatively well when they initiate treatment and highlights the need for further research as treatment programmes expand access to all HIV-positive people. 
A

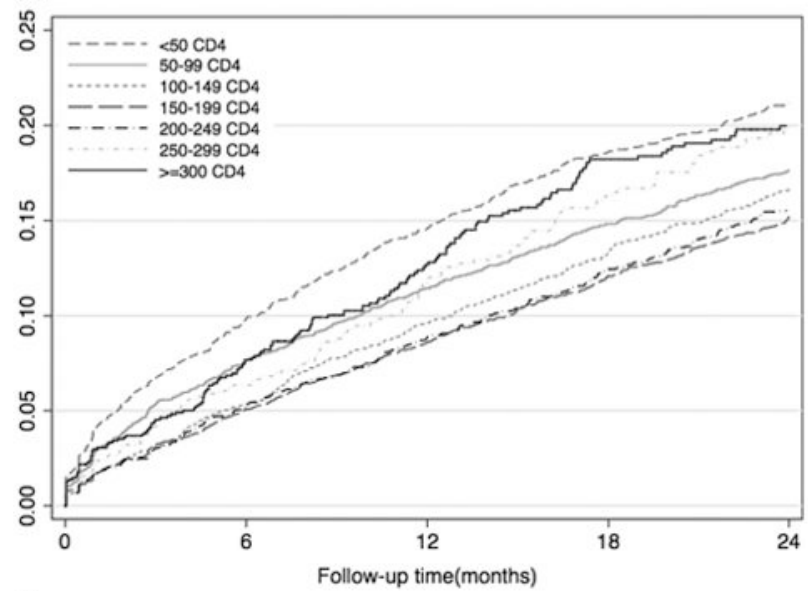

B

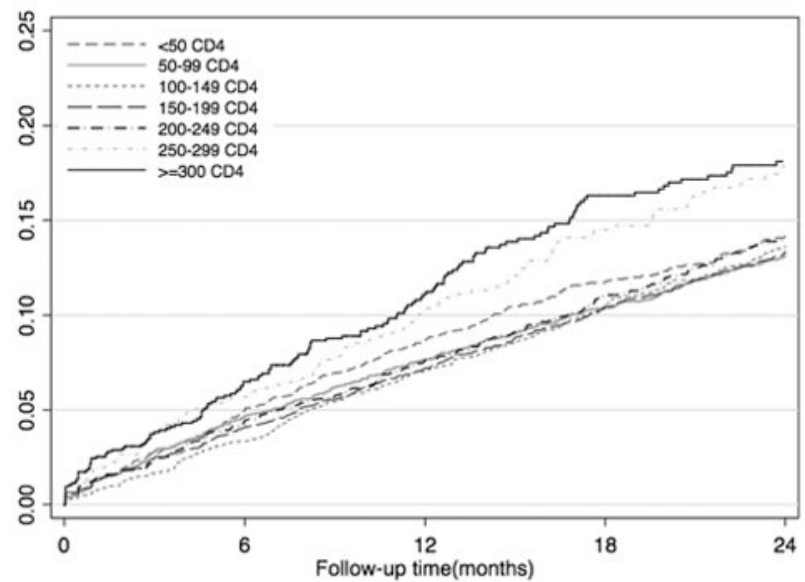

Figure 1.

Kaplan-Meier plots showing observed and corrected 24 months loss to follow-up (LTFU) by CD4 count at ART initiation. (A) Observed LTFU by CD4 count at antiretroviral therapy (ART) initiation 0-24 months follow-up. (B) Corrected LTFU by CD4 count at ART initiation 0-24 months follow-up. 


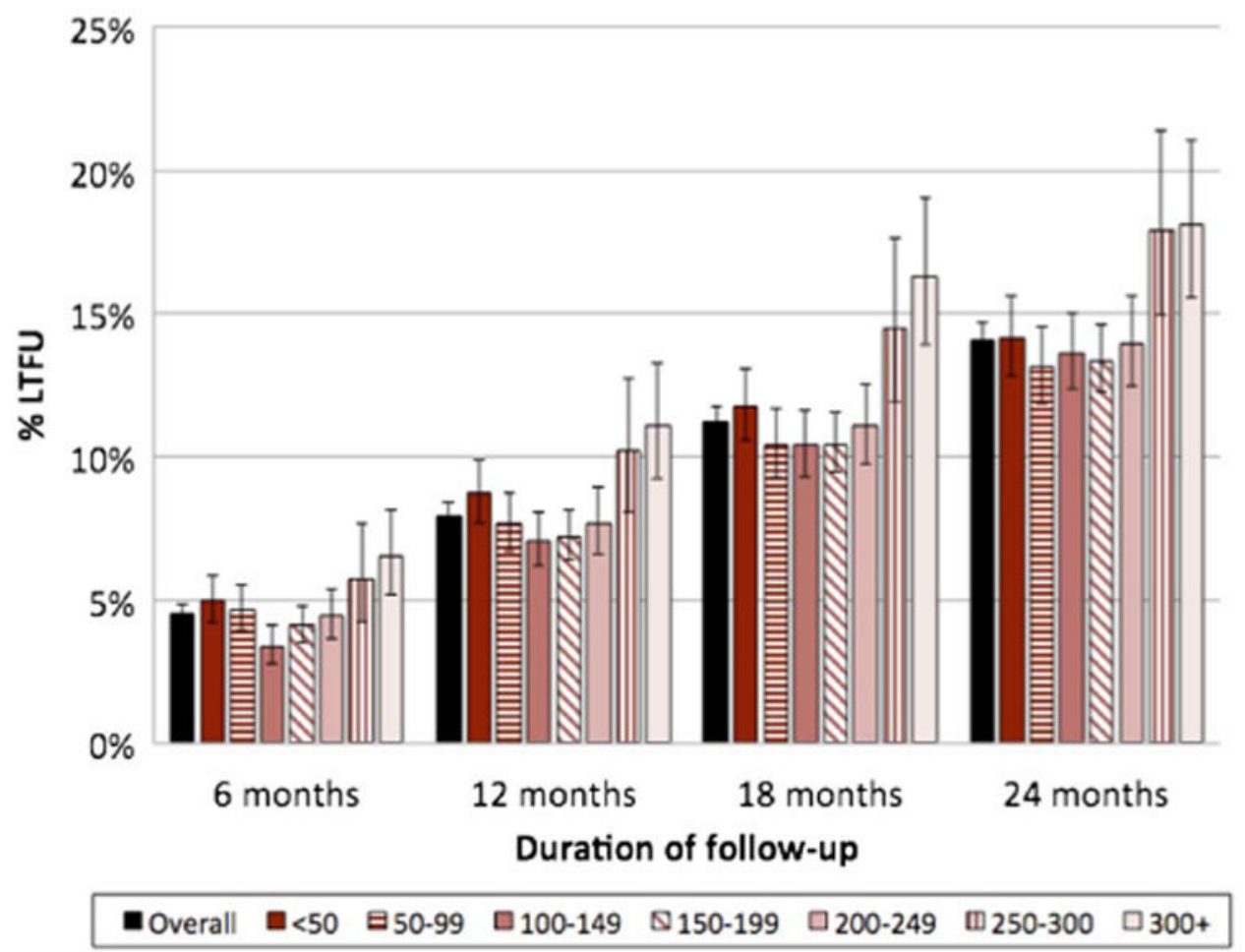

Figure 2.

Corrected loss to follow-up from Kaplan-Meier estimates by CD4 count at antiretroviral therapy initiation. 


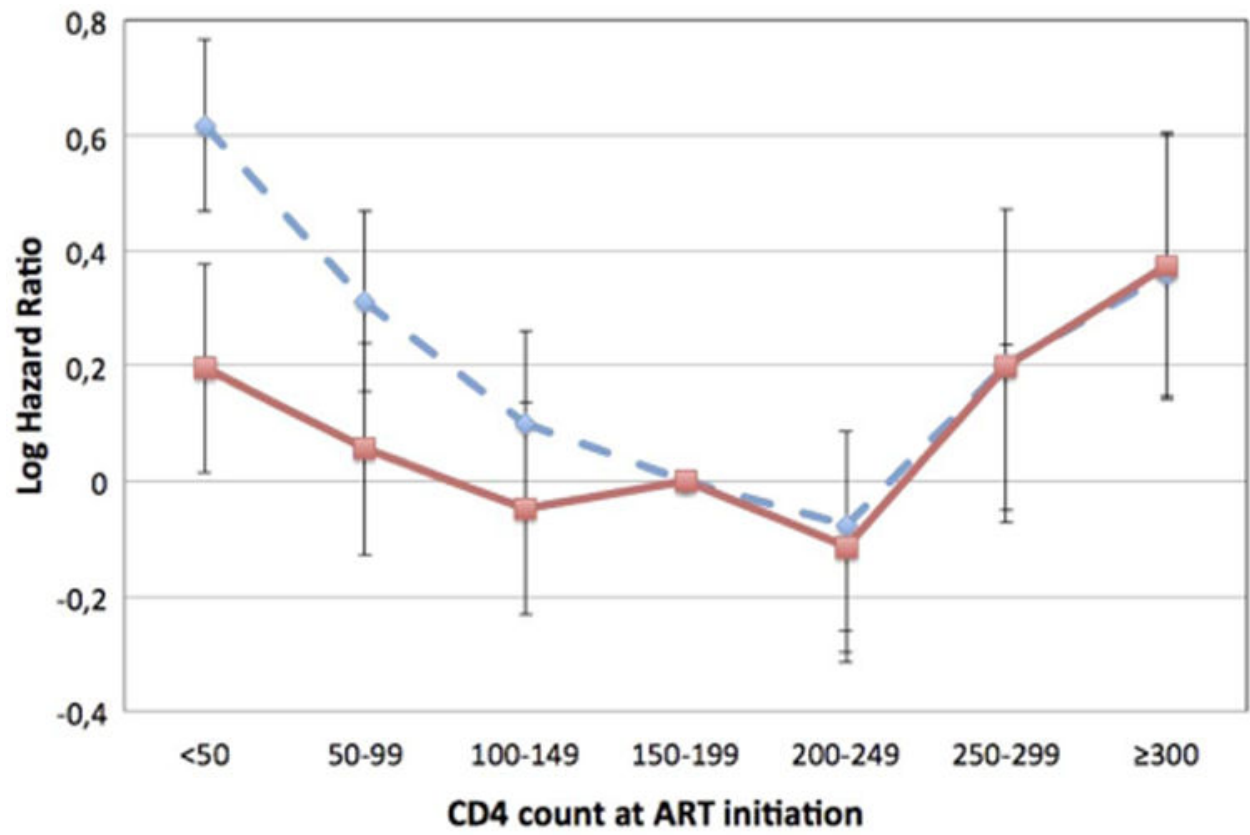

\section{$\Rightarrow$ Observed LTFU $\quad-$-Corrected LTFU}

Figure 3.

Adjusted 12-months log hazard ratios of observed and corrected loss to follow-up from Cox's proportional hazards models by CD4 count at antiretroviral therapy (ART) initiation. Adjusted for year of ART initiation, gender, age, programme size and rate of expansion. 


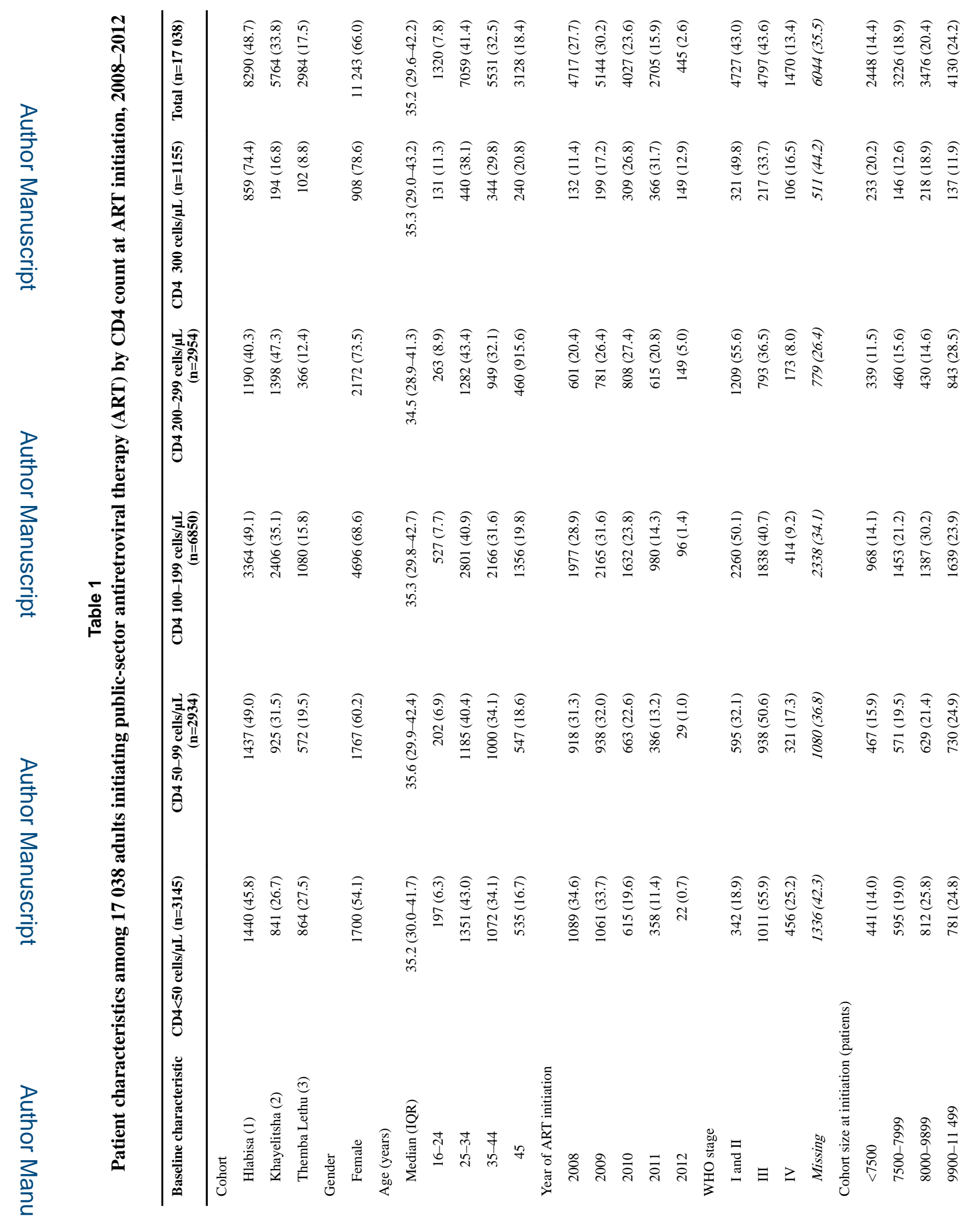

J Epidemiol Community Health. Author manuscript; available in PMC 2016 June 01. 
Grimsrud et al.

Page 17

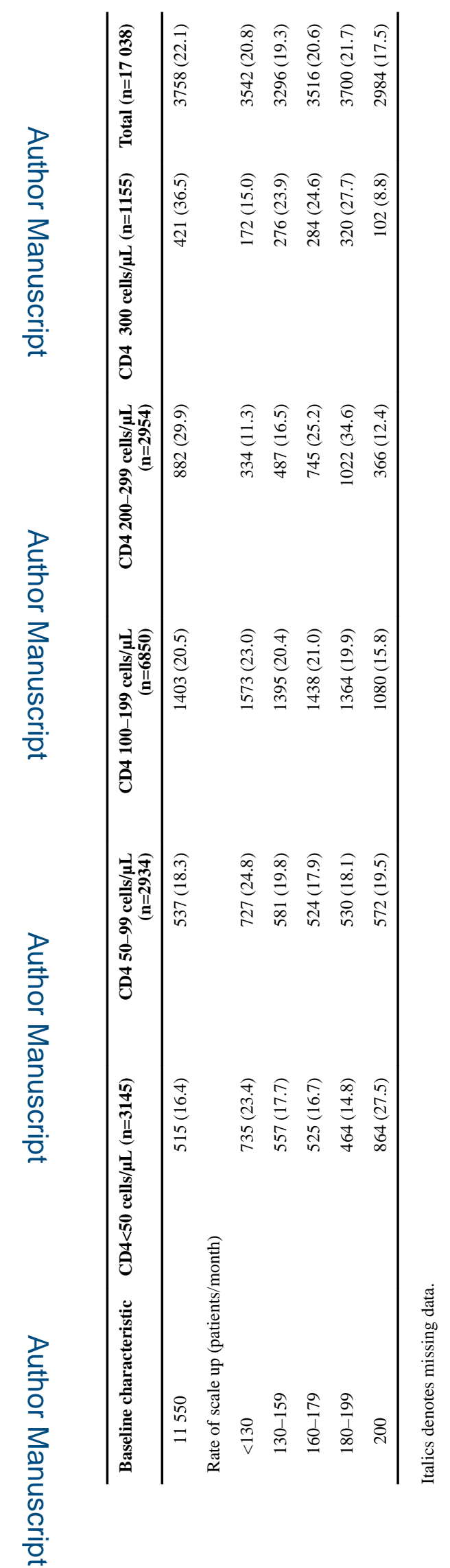

J Epidemiol Community Health. Author manuscript; available in PMC 2016 June 01. 
Table 2

HRs of overall, 0-3, 3-12 and 12-24 months corrected LTFU by CD4 count at ART initiation*

\begin{tabular}{|c|c|c|c|c|}
\hline & Overall (0-24 months) $n=17038$ & 0-3 months n=17038 & 3-12 months $n=15470$ & 12-24 months $n=12728$ \\
\hline \multicolumn{5}{|c|}{ Crude HR (cells/ $\mu \mathrm{L})$} \\
\hline$<50$ & $1.13(0.98$ to 1.30$)$ & $1.10(0.81$ to 1.49$)$ & $1.34(1.07$ to 1.68$)$ & $0.95(0.75$ to 1.20$)$ \\
\hline $50-99$ & $1.01(0.88$ to 1.17$)$ & $1.14(0.85$ to 1.54$)$ & $1.05(0.83$ to 1.33$)$ & $0.92(0.73$ to 1.15$)$ \\
\hline $100-149$ & $1.01(0.88$ to 1.17$)$ & $0.66(0.47$ to 0.94$)$ & $1.14(0.92$ to 1.43$)$ & $1.08(0.87$ to 1.33$)$ \\
\hline $150-199$ & $1.0(\mathrm{ref})$ & $1.0(\mathrm{ref})$ & 1.0 (ref) & $1.0(\mathrm{ref})$ \\
\hline $200-249$ & $0.95(0.82$ to 1.11$)$ & $0.84(0.60$ to 1.18$)$ & $1.03(0.81$ to 1.32$)$ & $0.94(0.74$ to 1.19$)$ \\
\hline $250-299$ & $1.38(1.11$ to 1.70$)$ & $1.56(1.01$ to 2.39$)$ & $1.39(0.98$ to 1.96$)$ & $1.27(0.89$ to 1.81$)$ \\
\hline 2300 & $1.62(1.25$ to 1.95$)$ & $1.95(1.35$ to 2.81$)$ & $1.70(1.27$ to 2.27$)$ & $1.34(0.97$ to 1.87$)$ \\
\hline \multicolumn{5}{|c|}{ Adjusted ${ }^{\dagger}-\mathrm{aHR}($ cells $/ \mu \mathrm{L})$} \\
\hline$<50$ & $1.08(0.94$ to 1.24$)$ & $1.07(0.79$ to 1.45$)$ & $1.30(1.04$ to 1.63$)$ & $0.89(0.70$ to 1.12$)$ \\
\hline $50-99$ & $0.98(0.85$ to 1.13$)$ & $1.12(0.83$ to 1.52$)$ & $1.02(0.81$ to 1.29$)$ & $0.88(0.70$ to 1.11$)$ \\
\hline $100-149$ & $1.00(0.87$ to 1.14$)$ & $0.66(0.47$ to 0.93$)$ & $1.12(0.90$ to 1.40$)$ & $1.06(0.85$ to 1.30$)$ \\
\hline $150-199$ & $1.0(\mathrm{ref})$ & $1.0(\mathrm{ref})$ & 1.0 (ref) & 1.0 (ref) \\
\hline $200-249$ & $0.89(0.77$ to 1.04$)$ & $0.80(0.57$ to 1.12$)$ & 0.95 (74 to 1.22$)$ & $0.90(0.71$ to 1.15$)$ \\
\hline $250-299$ & $1.18(0.95$ to 1.46$)$ & $1.40(0.90$ to 2.16$)$ & $1.14(0.80$ to 1.62$)$ & $1.12(0.89$ to 1.60$)$ \\
\hline$\geq 300$ & $1.35(1.12$ to 1.63$)$ & $1.68(1.15$ to 2.45$)$ & $1.35(1.00$ to 1.81$)$ & $1.17(0.84$ to 1.64$)$ \\
\hline
\end{tabular}

* All estimates are stratified by cohort.

${ }^{\dagger}$ Adjusted for year of ART initiation, gender, age, programme size and rate of expansion.

ART, antiretroviral therapy; LTFU, loss to follow-up. 